\title{
A New Lindley-Burr XII Distribution: Model, Properties and Applications
}

\author{
Boikanyo Makubate ${ }^{1}$, Broderick Oluyede ${ }^{1} \&$ Morongwa Gabanakgosi $^{1}$ \\ ${ }^{1}$ Department of Mathematics and Statistical Sciences, Botswana International University of Science and Technology, \\ Private Bag 16, Palapye, Botswana \\ Correspondence: Morongwa Gabanakgosi, Department of Mathematics and Statistical Sciences, Botswana International \\ University of Science and Technology, P. Bag 16, Palapye, Botswana
}

Received: October 27, 2020 Accepted: May 26, 2021 Online Published: June 1, 2021

doi:10.5539/ijsp.v10n4p33

URL: https://doi.org/10.5539/ijsp.v10n4p33

\begin{abstract}
A new distribution called the Lindley-Burr XII (LBXII) distribution is proposed and studied. Some structural properties of the new distribution including moments, conditional moments, distribution of the order statistics and Rényi entropy are derived. Maximum likelihood estimation technique is used to estimate the model parameters. A simulation study to examine the bias and mean square error of the maximum likelihood estimators is presented and applications to real data sets in order to illustrate the usefulness of the new distribution are given.
\end{abstract}

Keywords: Lindley distribution, Burr XII distribution, generalized distribution, monte Carlo simulations, maximum likelihood estimation

\section{Introduction}

There are several generalizations of the Lindley distribution that are considered to be useful life models, and are suitable for modeling data with different types of hazard rate functions including increasing, decreasing, bathtub and unimodal. (Lindley, 1958) used a mixture of exponential and length-biased exponential distributions to illustrate the difference between fiducial and posterior distributions. This mixture is called the Lindley (L) distribution. There are several generalizations of the Lindley distribution in the literature including the works by (Oluyede, Yang and Omolo, 2015) and (Nadarajah, Bakouch and Tahmasbi, 2011). (Oluyede and Yang, 2015) presented the beta generalized Lindley distribution. (Ghitany, Al-Mutairi, Balakrishnan and Al-Ezeni, 2013) presented a two-parameter power Lindley distribution. (Zakerzadeh and Dolati, 2009) studied an extension of the Lindley distribution. These models constitute flexible family of distributions in terms of the varieties of shapes and hazard functions. The cumulative distribution function (cdf) of the Lindley distribution is given by

$$
G_{L}(x ; \lambda)=1-\frac{\lambda+1+\lambda x}{\lambda+1} e^{-\lambda x}, \quad \text { for } x>0, \text { and } \lambda>0 .
$$

The corresponding Lindley probability density function (pdf) is given by

$$
g_{L}(x ; \lambda)=\frac{\lambda^{2}(1+x)}{1+\lambda} e^{-\lambda x}, \quad \text { for } x>0, \text { and } \lambda>0 .
$$

Lindley distribution is a combination of exponential and gamma distributions, that is $f(x ; \lambda)=(1-p) f_{G}(x ; \lambda)+p f_{E}(x ; \lambda)$ with $p=\frac{1}{1+\lambda}$, where $f_{G}(x ; \lambda) \equiv \operatorname{GAM}(2, \lambda)$, and $f_{E}(x ; \lambda) \equiv \operatorname{EXP}(\lambda)$. The cdf of the generalized Lindley (GL) distribution (Nadarajah et al., 2011) is given by

$$
G_{G L}(x ; \alpha, \lambda)=\left[1-\frac{1+\lambda+\lambda x}{1+\lambda} \exp (-\lambda x)\right]^{\alpha},
$$

and the corresponding pdf is given by

$$
g_{G L}(x ; \alpha, \lambda)=\frac{\alpha \lambda^{2}}{1+\lambda}(1+x)\left[1-\frac{1+\lambda+\lambda x}{1+\lambda} \exp (-\lambda x)\right]^{\alpha-1} \exp (-\lambda x)
$$

for $x>0, \lambda>0, \alpha>0$. This distribution is the exponentiated Lindley distribution.

We conisider the following generalization of the Lindley and Burr XII distributions. Let $X_{1}$ and $X_{2}$ be independent random variables with Lindley and Burr XII distributions, respectively. That is, $F_{X_{1}}(x)=1-\frac{\lambda+1+\lambda x}{\lambda+1} e^{-\lambda x}$, and $F_{X_{2}}(x)=1-\left(1+x^{c}\right)^{-k}$, 
for $x>0$, and $c, k>0$, respectively, then the distribution of $X=\min \left(X_{1}, X_{2}\right)$ is the Lindley-Burr XII (LBXII) distribution with parameters $\lambda>0, c>0$, and $k>0$. This model is good for modeling data with different types of hazard rate functions including increasing, decreasing and bathtub. The hazard rate function is also additive, see (Oluyede, Foya, WarahenaLiyanage and Huang, 2016). This paper employs the competing risk transformation involving both the Lindley and Burr XII distributions to obtain a new more flexible distribution to describe survival and reliability data. This transformation generalizes both the Lindley and Burr XII distribution. The combined distribution of Lindley and Burr XII is obtained from the product of the survival functions of the Lindley and Burr XII distributions via competing risk model. A motivation for developing this model is the advantages presented by this extended distribution with respect to having a hazard function that exhibits increasing, decreasing and bathtub shapes, as well as the versatility and flexibility of the Lindley and Burr XII distributions in modeling lifetime data.

This paper is organized as follows. In section 2, some basic results, the LBXII distribution and its sub-models, hazard rate and quantile functions are presented. The moments, conditional moments, mean and median deviations are given in section 3. Section 4 contain some additional useful results on the distribution of order statistics and Rényi entropy. In section 5, results on the estimation of the parameters of the LBXII distribution via the method of maximum likelihood are presented. A Monte Carlo simulation study is conducted to examine the bias and mean square error of the maximum likelihood estimators in section 6 . Applications are given in section 7, followed by some concluding remarks in section 8 .

\section{The Model, Sub-models, Hazard Rate and Quantile Functions}

In this section, the LBXII distribution, some sub-models, quantile function, hazard rate function as well some graphs are presented. The cdf and pdf of the LBXII distribution are given by

$$
G_{L B X I I}(x ; \lambda, c, k)=G(x ; \lambda, c, k)=1-\frac{1+\lambda+\lambda x}{1+\lambda} \frac{e^{-\lambda x}}{\left(1+x^{c}\right)^{k}},
$$

and

$$
g_{\text {LBXII }}(x ; \lambda, c, k)=\frac{\left(1+x^{c}\right)^{-k}}{1+\lambda} e^{-\lambda x}\left[\lambda^{2}(1+x)+\frac{k c x^{c-1}(1+\lambda+\lambda x)}{1+x^{c}}\right],
$$

for $\lambda, c, k>0$. If a random variable $X$ has the LBXII distribution, we write $X \sim \operatorname{LBXII}(\lambda, c, k)$. The parameters $c$ and $k$ are shape parameters and $\lambda$ is a scale parameter. Note that the LBXII pdf can be written as follows:

$$
g_{L B X I I}(x ; \lambda, c, k)=g_{L}(x ; \lambda) \bar{G}_{B}(x ; c, k)+\bar{G}_{L}(x ; \lambda) g_{B}(x ; c, k),
$$

where $\bar{G}_{B}(x ; c, k)=1-G(x ; c, k)$, and $\bar{G}_{L}(x ; \lambda)=1-G(x ; \lambda)$ are the survival functions of Burr XII and Lindley distributions, respectively, and $g_{L}(x ; \lambda), g_{B}(x ; c, k)$ are the pdf's of Lindley and Burr XII distributions.

Plots of the LBXII pdf shows different shapes including right skewed, almost symmetric and reverse J-shaped as indicated in Figure 1.

\subsection{Sub-models of LBXII Distribution}

In this subsection, we discuss some special models of the LBXII distribution.

- We obtain Lindley (L) distribution with $k=0$.

- When $\lambda=0$, we obtain the Burr XII (BXII) distribution.

- If $k=1$, we obtain Lindley-Log-logistic (LLLoG) distribution.

- If $k=1$, and $\lambda=0$, we obtain Log-logistic (LLoG) distribution.

- If $c=1$, we obtain Lindley-Lomax (LLomax) distribution.

- If $c=1$, and $\lambda=0$, we obtain Lomax (Lomax) distribution.

- If $c=k=1$, we have the one parameter distribution with cdf

$$
G(x ; \lambda)=1-\frac{1+\lambda+\lambda x}{1+\lambda} \frac{e^{-\lambda x}}{(1+x)},
$$

for $x>0$, and $\lambda>0$. 


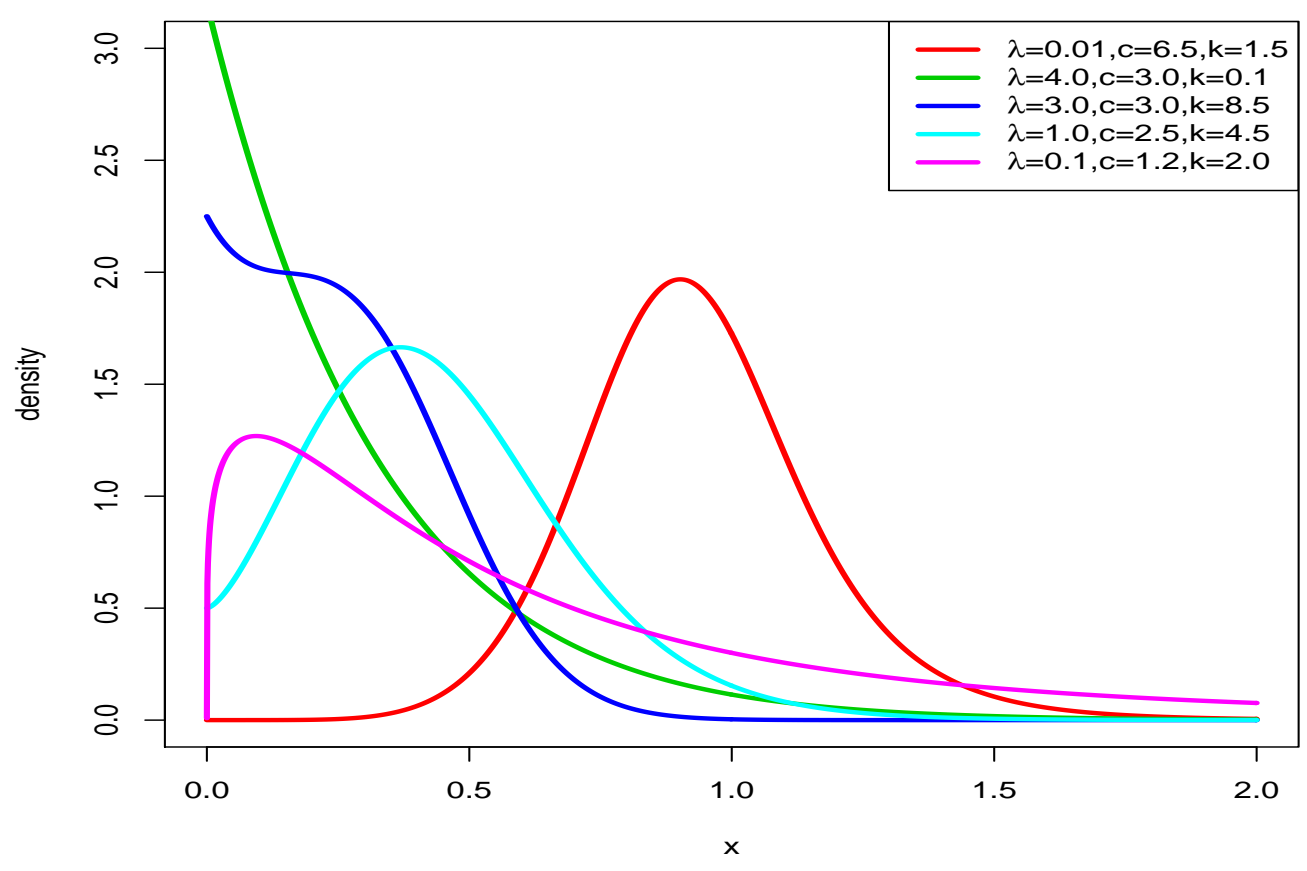

Figure 1. Plots of LBXII Density Function

\subsection{Hazard Rate and Quantile Functions}

In this section, we present the hazard rate and quantile functions of the LBXII distribution. We note from equation (7) that the hazard rate function of the LBXII distribution is given by

$$
h_{G}(x)=h_{G_{L}}(x)+h_{G_{B}}(x),
$$

where $h_{G_{L}}(x)$ and $h_{G_{B}}(x)$ are the hazard rate functions of the Lindley and Burr XII distributions, respectively, that is,

$$
h_{G}(x)=\frac{g(x)}{\bar{G}(x)}=\frac{\lambda^{2}(1+x)}{1+\lambda+\lambda x}+\left(1+x^{c}\right)^{-1} k c x^{c-1} .
$$

Plots of the LBXII hazard rate function shows different shapes including decreasing, increasing, as well as upside down bathtub shapes as shown in Figure 2. The quantile function of the LBXII distribution is obtained by solving the non-linear equation:

$$
G(x ; \lambda, c, k)=1-\frac{1+\lambda+\lambda x}{1+\lambda} \frac{e^{-\lambda x}}{\left(1+x^{c}\right)^{k}}=u,
$$

$0 \leq u \leq 1$, that is,

$$
\lambda x+k \log \left(1+x^{c}\right)-\log \left(1+\frac{\lambda x}{1+\lambda}\right)+\log (1-u)=0 .
$$

Thus, random numbers can be readily generated from the LBXII distribution by numerically solving the non-linear equation (12). Quantiles of the LBXII distribution are given in Table 1. 


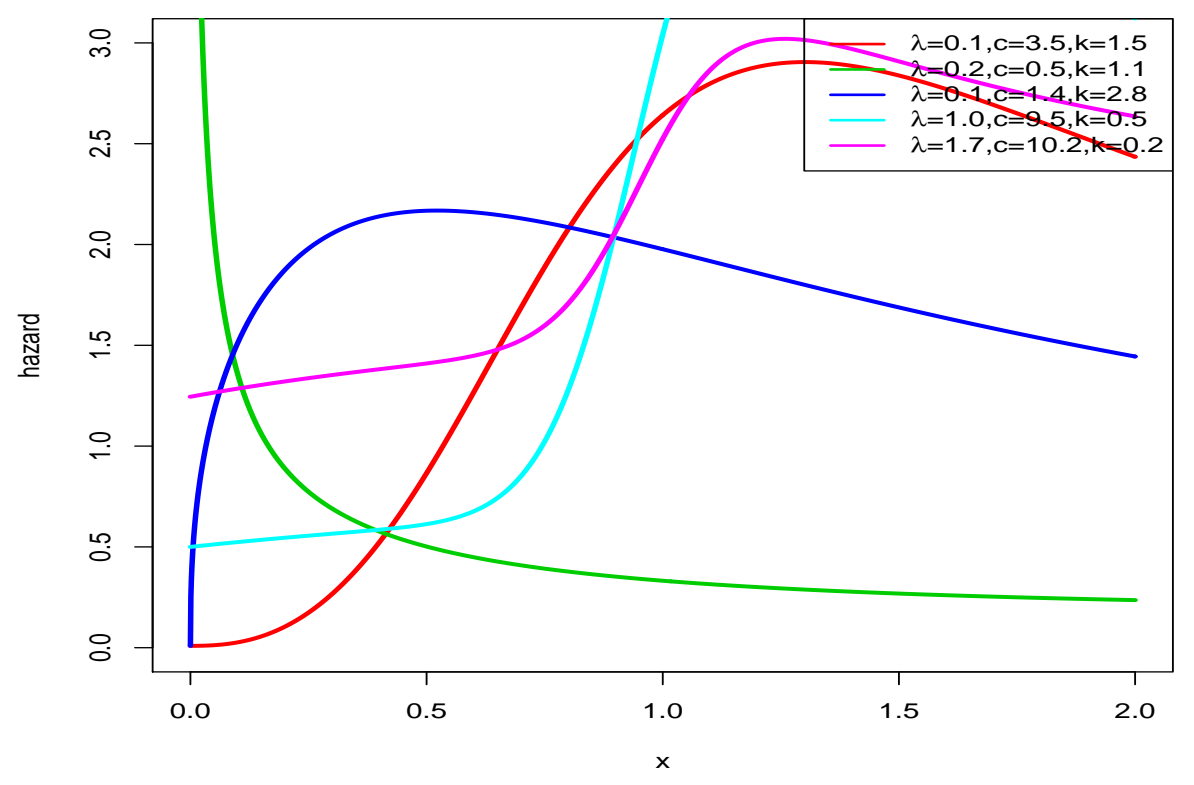

Figure 2. Plots of LBXII Hazard Rate Function

Table 1. Table of Quantile for LBXII Distribution

\begin{tabular}{cccccc}
\hline \multicolumn{5}{c}{$(\lambda, c, k)$} \\
\hline$u$ & $(0.9,3.5,5.5)$ & $(0.7,1,1.5)$ & $(1.3,2,4.5)$ & $(3.1,4.5,3.5)$ & $(1.2,2.5,5.5)$ \\
\hline 0.1 & 0.1953 & 0.0602 & 0.0911 & 0.0447 & 0.1179 \\
0.2 & 0.3037 & 0.1306 & 0.1544 & 0.0941 & 0.1943 \\
0.3 & 0.3787 & 0.2143 & 0.2106 & 0.1494 & 0.2563 \\
0.4 & 0.4406 & 0.3160 & 0.2652 & 0.2116 & 0.3128 \\
0.5 & 0.4972 & 0.4434 & 0.3214 & 0.2822 & 0.3681 \\
0.6 & 0.5529 & 0.6093 & 0.3825 & 0.3620 & 0.4256 \\
0.7 & 0.6121 & 0.8387 & 0.4537 & 0.4519 & 0.4897 \\
0.8 & 0.6814 & 1.1894 & 0.5448 & 0.5551 & 0.5681 \\
0.9 & 0.7787 & 1.8529 & 0.6866 & 0.6867 & 0.6839 \\
\hline
\end{tabular}

\section{Moments, Conditional Moments and Mean Deviations}

In this section, we present the moments, conditional moments and mean deviations of the LBXII distribution.

\subsection{Moments}

The $r^{\text {th }}$ moment of the LBXII distribution is given by

$$
\begin{aligned}
E\left(X^{r}\right) & =r \int_{0}^{\infty} x^{r-1} \frac{1+\lambda+\lambda x}{1+\lambda} \frac{e^{-\lambda x}}{\left(1+x^{c}\right)^{k}} d x \\
& =\sum_{s=0}^{\infty} \frac{r(-1)^{s} \lambda^{s}}{s !} \int_{0}^{\infty} x^{r+s-1}\left(1+\frac{\lambda x}{1+\lambda}\right)\left(1+x^{c}\right)^{-k} d x \\
& =\sum_{s=0}^{\infty} \frac{r(-1)^{s} \lambda^{s}}{s ! c}\left[\int_{0}^{1} t^{k-1-\frac{1}{c}-\frac{r+s-1}{c}}(1-t)^{\frac{r+s-1}{c}+\frac{1}{c}-1} d t\right. \\
& \left.+\frac{\lambda}{1+\lambda} \int_{0}^{\infty} t^{k-1-\frac{1}{c}-\frac{r+s}{c}}(1-t)^{\frac{r+s+1}{c}-1} d t\right] \\
& =\sum_{s=0}^{\infty} \frac{r(-1)^{s} \lambda^{s}}{s ! c}\left[B\left(k-\frac{r+s}{c}, \frac{r+s}{c}\right)+\frac{\lambda}{1+\lambda} B\left(k-\frac{r+s+1}{c}, \frac{r+s+1}{c}\right)\right],
\end{aligned}
$$


where we have applied the transformation $t=\left(1+x^{c}\right)^{-1}$, and $B(a, b)=\int_{0}^{1} t^{a-1}(1-t)^{b-1} d t$ is the complete beta function. The coefficients of variation (CV), Skewness (CS) and Kurtosis (CK) can be readily obtained. The variance $\left(\sigma^{2}\right)$, Standard deviation $(\mathrm{SD}=\sigma)$, coefficient of variation $(\mathrm{CV})$, coefficient of skewness (CS) and coefficient of kurtosis $(\mathrm{CK})$ are given by

$$
\begin{gathered}
\sigma^{2}=\mu_{2}^{\prime}-\mu^{2}, \quad C V=\frac{\sigma}{\mu}=\frac{\sqrt{\mu_{2}^{\prime}-\mu^{2}}}{\mu}=\sqrt{\frac{\mu_{2}^{\prime}}{\mu^{2}}-1}, \\
C S=\frac{E\left[(X-\mu)^{3}\right]}{\left[E(X-\mu)^{2}\right]^{3 / 2}}=\frac{\mu_{3}^{\prime}-3 \mu \mu_{2}^{\prime}+2 \mu^{3}}{\left(\mu_{2}^{\prime}-\mu^{2}\right)^{3 / 2}},
\end{gathered}
$$

and

$$
C K=\frac{E\left[(X-\mu)^{4}\right]}{\left[E(X-\mu)^{2}\right]^{2}}=\frac{\mu_{4}^{\prime}-4 \mu \mu_{3}^{\prime}+6 \mu^{2} \mu_{2}^{\prime}-3 \mu^{4}}{\left(\mu_{2}^{\prime}-\mu^{2}\right)^{2}},
$$

respectively. Some moments for selected parameter values for the LBXII distribution are given in Table 2 and 3D plots for skewness and kurtosis are presented in Figure 3, Figure 4 and Figure 5, respectively. The 3D plots shows the dependence of skewness and kurtosis on the shape parameters.

Table 2. Table of Moments for LBXII Distribution

\begin{tabular}{cccccc}
\hline \multicolumn{5}{c}{$(\lambda, c, k)$} \\
\hline Moments & $(0.5,0.5,0.5)$ & $(4.5,2.7,1.0)$ & $(1.5,1.5,0.2)$ & $(2.0,2.0,3.5)$ & $(3.4,4.0,0.5)$ \\
\hline$E X$ & 0.1399 & 0.2347 & 0.2942 & 0.3178 & 0.2912 \\
$E X^{2}$ & 0.0843 & 0.0970 & 0.1789 & 0.1505 & 0.1455 \\
$E X^{3}$ & 0.0604 & 0.0526 & 0.1266 & 0.0872 & 0.0907 \\
$E X^{4}$ & 0.0471 & 0.0334 & 0.0973 & 0.0574 & 0.0638 \\
$E X^{5}$ & 0.0386 & 0.0235 & 0.0787 & 0.0412 & 0.0485 \\
$E X^{6}$ & 0.0327 & 0.0177 & 0.0660 & 0.0314 & 0.0387 \\
SD & 0.0284 & 0.0139 & 0.0568 & 0.0251 & 0.0321 \\
CV & 0.0251 & 0.0115 & 0.0498 & 0.0207 & 0.0273 \\
CS & 0.0224 & 0.0098 & 0.0443 & 0.0176 & 0.0236 \\
CK & 0.0203 & 0.0083 & 0.0398 & 0.0152 & 0.0209 \\
\hline
\end{tabular}

$\operatorname{LBXII}(\lambda, c, 2.75)$

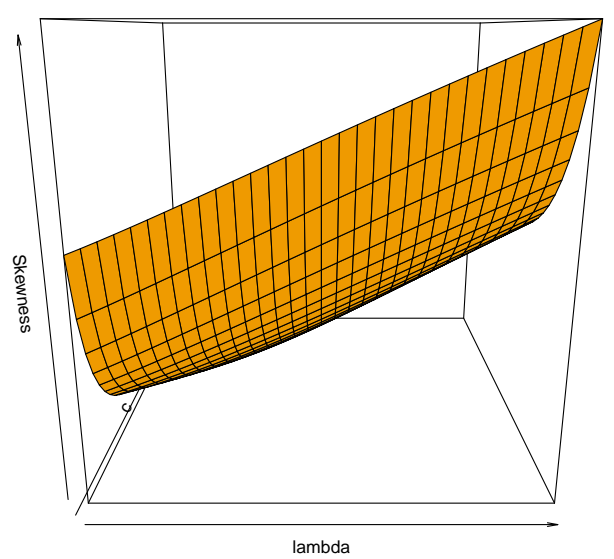

$\operatorname{LBXII}(\lambda, c, 2.75)$

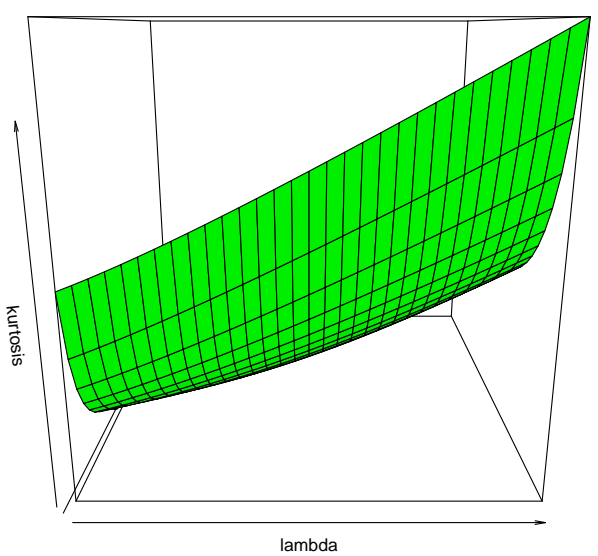

Figure 3. Plots of Skewness and Kurtosis for LBXII distribution 
$\operatorname{LBXII}(0.05, \mathrm{c}, \mathrm{k})$

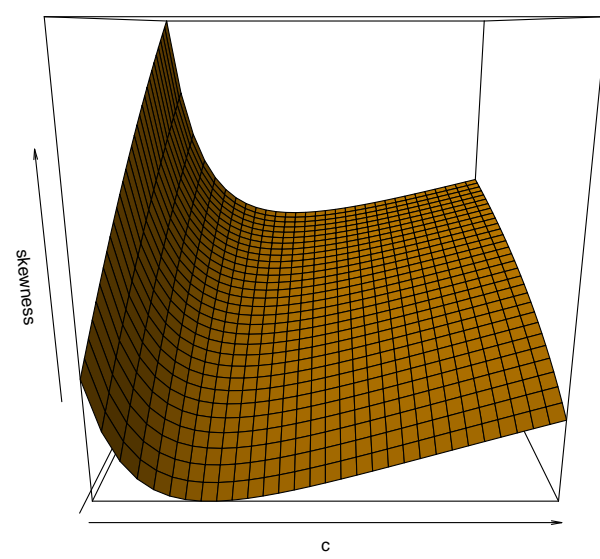

$\operatorname{LBXII}(0.05, \mathrm{c}, \mathrm{k})$

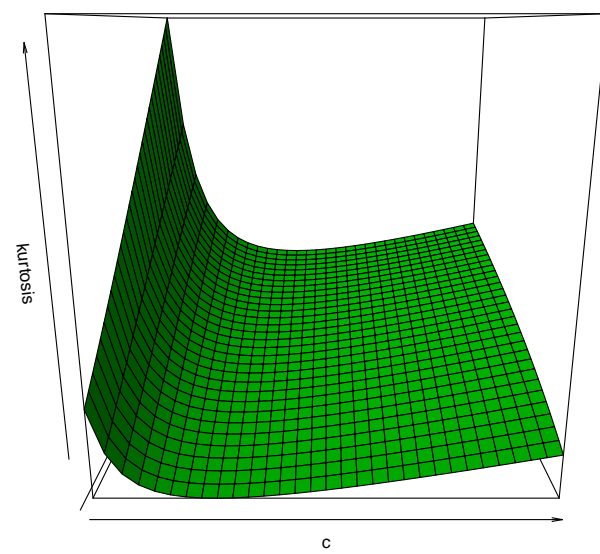

Figure 4. Plots of Skewness and Kurtosis for LBXII distribution

$\operatorname{LBXII}(\lambda, 1.5, \mathrm{k})$

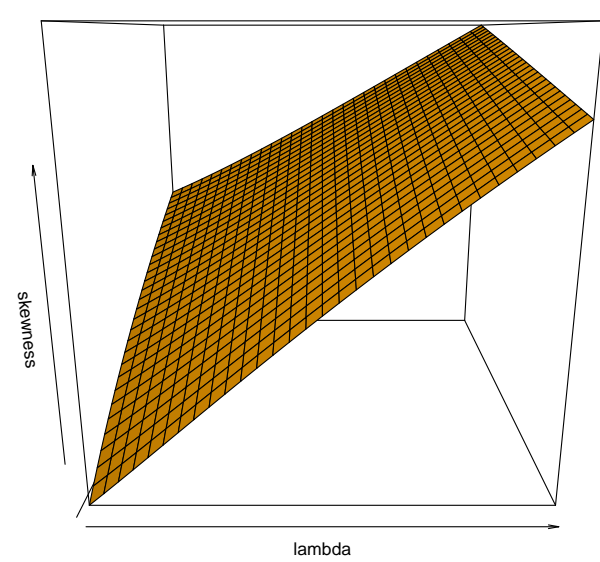

$\operatorname{LBXII}(\lambda, 1, \mathrm{k})$

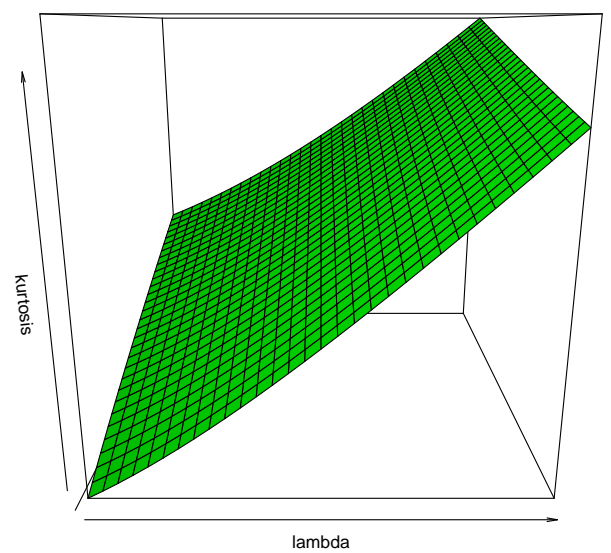

Figure 5. Plots of Skewness and Kurtosis for LBXII distribution

\subsection{Conditional Moments}

In this subsection, the $r^{\text {th }}$ conditional moment is presented. The mean residual life function as well as income inequality measures such as Bonferroni and Lorenz curves as well as mean deviation about the mean and mean deviation about the 
median can be readily obtained from the conditional moments. The $r^{\text {th }}$ conditional moment is given by

$$
\begin{aligned}
E\left(X^{r} \mid X>t\right) & =\frac{1}{\bar{G}(t)} \int_{t}^{\infty} x^{r} \frac{\left(1+x^{c}\right)^{-k}}{1+\lambda} e^{-\lambda x}\left[\lambda^{2}(1+x)+\frac{(1+\lambda+\lambda x) k c x^{c-1}}{1+x^{c}}\right] d x \\
& =\frac{1}{\bar{G}(t)} \sum_{s=0}^{\infty} \frac{(-1)^{s} \lambda^{s}}{s !} \int_{t}^{\infty} x^{r+s} \frac{\left(1+x^{c}\right)^{-k}}{1+\lambda}\left[\lambda^{2}(1+x)+\frac{(1+\lambda+\lambda x) k c x^{c-1}}{1+x^{c}}\right] d x \\
& =\frac{1}{\bar{G}(t)} \sum_{s=0}^{\infty} \frac{(-1)^{s} \lambda^{s}}{s !}\left(\frac{\lambda^{2}}{1+\lambda} \int_{t}^{\infty} x^{r+s}\left(1+x^{c}\right)^{-k}(1+x) d x\right. \\
& \left.+k c \int_{t}^{\infty} x^{r+s+c-1}\left(1+\frac{\lambda x}{1+\lambda}\right)\left(1+x^{c}\right)^{-k-1} d x\right) \\
& =\frac{1}{\bar{G}(t)} \sum_{s=0}^{\infty} \frac{(-1)^{s} \lambda^{s}}{s ! c}\left[\frac { \lambda ^ { 2 } } { 1 + \lambda } \left(B_{\left(1+t^{c}\right)^{-1}}\left(k-\frac{r+s+1}{c}, \frac{r+s+1}{c}\right)\right.\right. \\
& \left.+B_{\left(1+t^{c}\right)^{-1}}\left(k-\frac{r+s+2}{c}, \frac{r+s+2}{c}\right)\right) \\
& +k c\left(B_{\left(1+t^{c}\right)^{-1}}\left(k+1-\frac{r+s+c}{c}, \frac{r+s+c}{c}\right)\right. \\
& \left.\left.+\frac{\lambda}{1+\lambda} B_{\left(1+t^{c}\right)^{-1}}\left(k-\frac{r+s+c+1}{c}, \frac{r+s+c+1}{c}\right)\right)\right]
\end{aligned}
$$

where $B_{y}(a, b)=\int_{0}^{y} t^{a-1}(1-t)^{b-1} d t$ is the incomplete beta function.

\subsection{Mean Deviation, Lorenz and Bonferroni Curves}

Mean deviation about the mean and mean deviation about the median as well as Lorenz and Bonferroni Curves for the LBXII distribution are presented in this subsection. The application of Lorenz and Bonferroni curves is not limited to economics for the study of income and poverty, but they can also be used to other field like reliability, demography, insurance and medicine.

\subsubsection{Mean Deviations}

The mean deviation about the mean and the mean deviation about the median are defined by

$$
\delta_{1}(x)=\int_{0}^{\infty}|x-\mu| g_{L B X I I}(x) d x \quad \text { and } \quad \delta_{2}(x)=\int_{0}^{\infty}|x-M| g_{L B X I I}(x) d x,
$$

respectively, where $\mu=E[X]$ and $M=\operatorname{Median}(X)$ denotes the mean and median, respectively. We note that $\delta_{1}(x)$ and $\delta_{2}(x)$ can be expressed as $\delta_{1}(x)=2 \mu G_{L B X I I}(\mu)-2 \mu+2 \int_{\mu}^{\infty} g_{L B X I I}(x) d x$ and $\delta_{2}(x)=-\mu+2 \int_{M}^{\infty} x g_{L B X I I}(x) d x$, respectively, that is,

$$
\delta_{1}(x)=2 \mu G_{L B X I I}(\mu)-2 \mu+2 T(\mu) \quad \text { and } \quad \delta_{2}(x)=2 T(M)-\mu,
$$

where

$$
\begin{aligned}
T(\mu) & =\int_{\mu}^{\infty} x g_{L B X I I}(x) d x \\
& =\sum_{s=0}^{\infty} \frac{(-1)^{s} \lambda^{s}}{s ! c}\left[\frac{\lambda^{2}}{1+\lambda}\left(B_{\left(1+\mu^{c}\right)^{-1}}\left(k-\frac{s+2}{c}, \frac{s+2}{c}\right)+B_{\left(1+\mu^{c}\right)^{-1}}\left(k-\frac{s+3}{c}, \frac{s+3}{c}\right)\right)\right. \\
& \left.+k c\left(B_{\left(1+\mu^{c}\right)^{-1}}\left(k+1-\frac{1+s+c}{c}, \frac{1+s+c}{c}\right)+\frac{\lambda}{1+\lambda} B_{\left(1+\mu^{c}\right)^{-1}}\left(k-\frac{s+c+2}{c}, \frac{s+c+2}{c}\right)\right)\right] .
\end{aligned}
$$

\subsubsection{Bonferroni and Lorenz Curves}

Bonferroni and Lorenz curves for the LBXII didtribution are given as

$B(p)=\frac{1}{p \mu} \int_{0}^{q} x g_{L B X I I}(x) d x=\frac{1}{p \mu}[\mu-T(q)]$, and $L(p)=\frac{1}{\mu} \int_{0}^{q} x g_{L B X I I}(x) d x=\frac{1}{\mu}[\mu-T(q)]$, respectively, where $T(q)=\int_{q}^{\infty} x g_{L B X I I}(x) d x, q=G_{L B X I I}^{-1}(p), 0 \leq p \leq 1$. 


\section{Order Statistics and Rényi Entropy}

In this section, we present the pdf of the $i^{\text {th }}$ order statistic from the LBXII distribution as well as Rényi entropy.

\subsection{Order Statistics}

Suppose that $X_{1}, \cdots, X_{n}$ is a random sample of size $n$ from a continuous pdf, $g(x)$. Let $X_{1: n}<X_{2: n}<\cdots<X_{n: n}$ denote the corresponding order statistics. If $X_{1}, \cdots, X_{n}$ is a random sample from LBXII distribution, it follows from the equations (5) and (6) that the pdf of the $i^{\text {th }}$ order statistic from the LBXII distribution is given by

$$
\begin{aligned}
g_{i: n}(x) & =\frac{n ! g_{L B X I I}(x)}{(i-1) !(n-i) !}\left[G_{L B X I I}(x)\right]^{i-1}\left[1-G_{L B X I I}(x)\right]^{n-i} \\
& =\frac{n ! g_{L B X I I}(x)}{(i-1) !(n-i) !} \sum_{s=0}^{n-i}\left(\begin{array}{c}
n-i \\
s
\end{array}\right)(-1)^{s}\left[G_{L B X I I}(x)\right]^{s+i-1} \\
& =\frac{n !}{(i-1) !(n-i) !} \sum_{s=0}^{n-i}\left(\begin{array}{c}
n-i \\
s
\end{array}\right) \frac{(-1)^{s}}{s+i} \frac{d}{d x}\left[G_{L B X X I}(x)\right]^{s+i},
\end{aligned}
$$

since $\left[G_{L B X I I}(x)\right]^{s+i-1} g_{L B X I I}(x)=\frac{1}{s+i} \frac{d}{d x}\left[G_{L B X I I}(x)\right]^{s+i}$. Therefore, the cdf of the $i^{t h}$ order statistic is given by

$$
G_{i: n}(x ; \lambda, c, k)=\frac{n !}{(i-1) !(n-i) !} \sum_{s=0}^{n-i}\left(\begin{array}{c}
n-i \\
s
\end{array}\right) \frac{(-1)^{s}}{s+i}\left[G_{L B X I I}(x ; \lambda, c, k)\right]^{s+i}
$$

where

$$
G_{L B X I I}(x ; \lambda, c, k)=1-\frac{1+\lambda+\lambda x}{1+\lambda} \frac{e^{-\lambda x}}{\left(1+x^{c}\right)^{k}},
$$

$\lambda, c, k>0$.

Consequently, the pdf of the $i^{t h}$ order statistic from the LBXII distribution is a linear combination of the exponentiated LBXII (ELBXII) pdf's with parameters: $s+i>0, \lambda>0, c>0$, and $k>0$.

\subsection{Rényi Entropy}

Rényi entropy (Rényi, 1960) is an extension of Shannon entropy. Rényi entropy is defined to be

$$
I_{R}(v)=\frac{1}{1-v} \log \left(\int_{0}^{\infty}\left[g_{L B X I I}(x ; \lambda, c, k)\right]^{v} d x\right), v \neq 1, v>0 .
$$

Rényi entropy tends to Shannon entropy as $v \rightarrow 1$. Note that

$$
\begin{aligned}
\int_{0}^{\infty} g_{L B X I I}^{v}(x) d x & =\int_{0}^{\infty} \frac{\left(1+x^{c}\right)^{v k}}{(1+\lambda)^{v}} \sum_{s, p=0}^{\infty} \frac{(-1)^{s}(v \lambda)^{s} x^{s}}{s !}\left(\begin{array}{l}
v \\
p
\end{array}\right)\left(\frac{1+\lambda+\lambda x}{1+x^{c}} k c x^{c-1}\right)^{p} \\
& \times\left(\lambda^{2}(1+x)\right)^{v-p} d x \\
& =\sum_{s, p, t, q=0}^{\infty} \frac{(-1)^{s}(v \lambda)^{s}(k c)^{p} \lambda^{2(v-p)+t}(1+\lambda)^{p-t}}{s !(1+\lambda)^{v}}\left(\begin{array}{l}
v \\
p
\end{array}\right)\left(\begin{array}{l}
p \\
t
\end{array}\right)\left(\begin{array}{c}
v-p \\
q
\end{array}\right) \\
& \times \int_{0}^{\infty} x^{s+c p-p+t+q}\left(1+x^{c}\right)^{-v k-p} d x \\
& =\sum_{s, p, t, q=0}^{\infty} \frac{(-1)^{s}(v \lambda)^{s}(k c)^{p} \lambda^{2(v-p)+t}(1+\lambda)^{p-t}}{s !(1+\lambda)^{v}}\left(\begin{array}{l}
v \\
p
\end{array}\right)\left(\begin{array}{l}
p \\
t
\end{array}\right)\left(\begin{array}{c}
v-p \\
q
\end{array}\right) \\
& \times \frac{1}{c} \int_{0}^{\infty} y^{\left.v k-\frac{1}{c}(s+t+q+1)-1\right)(1-y)^{\frac{1}{c}(s+t+q+1)-1} d y} \\
& =\sum_{s, p, t, q=0}^{\infty} \frac{(-1)^{s}(v \lambda)^{s}(k c)^{p} \lambda^{2(v-p)+t}(1+\lambda)^{p-t}}{s !(1+\lambda)^{v}}\left(\begin{array}{l}
v \\
p
\end{array}\right)\left(\begin{array}{l}
p \\
t
\end{array}\right)\left(\begin{array}{c}
v-p \\
q
\end{array}\right) \\
& \times \frac{1}{c} B\left(v k-\frac{1}{c}(s+t+q+1), \frac{1}{c}(s+t+q+1)\right),
\end{aligned}
$$


where $B(a, b)=\int_{0}^{1} t^{a-1}(1-t)^{b-1} d t$ is the complete beta function. Consequently, Rényi entropy for the LBXII distribution is given by

$$
\begin{aligned}
I_{R}(v) & =\frac{1}{1-v} \log \left[\sum_{s, p, t, q=0}^{\infty} \frac{(-1)^{s}(v \lambda)^{s}(k c)^{p} \lambda^{2(v-p)+t}(1+\lambda)^{p-t}}{s !(1+\lambda)^{v}}\left(\begin{array}{l}
v \\
p
\end{array}\right)\left(\begin{array}{c}
p \\
t
\end{array}\right)\left(\begin{array}{c}
v-p \\
q
\end{array}\right)\right. \\
& \left.\times \frac{1}{c} B\left(v k-\frac{(s+t+q+1)}{c}, \frac{(s+t+q+1)}{c}\right)\right], \quad v \neq 1, v>0 .
\end{aligned}
$$

\section{Maximum Likelihood Estimates}

The maximum likelihood estimates (MLEs) of the parameters of the LBXII distribution are presented in this section. Let $X_{i} \sim \operatorname{LBXII}(\lambda, c, k)$ and $\Delta=(\lambda, c, k)^{T}$ be the parameter vector. The log-likelihood $\ell=\ell(\Delta)$ based on a random sample of size $\mathrm{n}$ is given by

$$
\ell=\ell(\Delta)=-k \sum_{i=1}^{n} \ln \left(1+x_{i}^{c}\right)-n \ln (1+\lambda)-\lambda \sum_{i=1}^{n} x_{i}+\sum_{i=1}^{n} \ln \left[\lambda^{2}\left(1+x_{i}\right)+\frac{\left(1+\lambda+\lambda x_{i}\right) k c x_{i}^{c-1}}{1+x_{i}^{c}}\right] .
$$

Elements of the score vector $U=\left(\frac{\partial \ell}{\partial k}, \frac{\partial \ell}{\partial \lambda}, \frac{\partial \ell}{\partial c}\right)$ are given by:

$$
\begin{aligned}
& \frac{\partial \ell}{\partial k}=-\sum_{i=1}^{n} \ln \left(1+x_{i}^{c}\right)+\sum_{i=1}^{n}\left(\frac{\left(1+\lambda+\lambda x_{i}\right) c x_{i}^{c-1}}{\left.\lambda^{2}\left(1+x_{i}\right)+\frac{\left(1+\lambda+\lambda x_{i}\right) k c x_{i}^{c-1}}{1+x_{i}^{c}}\right),}\right. \\
& \frac{\partial \ell}{\partial \lambda}=-\frac{1}{1+\lambda}-\sum_{i=1}^{\infty} x_{i}+\sum_{i=1}^{n}\left(\frac{2 \lambda\left(1+x_{i}\right)+\frac{\left(1+x_{i}\right) k x_{i}^{c-1}}{1+x_{i}}}{\lambda^{2}\left(1+x_{i}\right)+\frac{\left(1+\lambda+\lambda x_{i}\right) c c_{i}^{c-1}}{1+x_{i}^{c}}}\right),
\end{aligned}
$$

and

$$
\begin{aligned}
\frac{\partial \ell}{\partial c} & =-k \sum_{i=1}^{n} \frac{x_{i}^{c} \ln \left(x_{i}\right)}{1+x_{i}^{c}}+\sum_{i=1}^{n}\left(\frac{\left[k\left(1+\lambda+\lambda x_{i}\right)\left[c x_{i}^{c-1}(-1)\left(1+x_{i}^{c}\right)^{-2} x_{i}^{c} \ln \left(x_{i}\right)\right]\right.}{\left(\lambda^{2}\left(1+x_{i}\right)+\frac{\left(1+\lambda+\lambda x_{i}\right) k c x_{i}^{c-1}}{1+x_{i}^{c}}\right)}\right. \\
& \left.+\frac{k\left(1+\lambda+\lambda x_{i}\right)\left(1+x_{i}^{c}\right)^{-1}\left[x_{i}^{c-1}+c x_{i}^{c-1} \ln \left(x_{i}\right)\right]}{\left(\lambda^{2}\left(1+x_{i}\right)+\frac{\left(1+\lambda+\lambda x_{i}\right) k c x_{i}^{c-1}}{1+x_{i}^{c}}\right)}\right)
\end{aligned}
$$

respectively. The equations obtained by setting the above partial derivatives to zero are not in closed form and the values of the parameters $k, \lambda, c$ must be found via iterative methods. The maximum likelihood estimates of the parameters, denoted by $\hat{\boldsymbol{\Delta}}$ is obtained by solving the non-linear equation $\left(\frac{\partial \ell}{\partial k}, \frac{\partial \ell}{\partial \lambda}, \frac{\partial \ell}{\partial c}\right)^{T}=\mathbf{0}$, using a numerical method such as NewtonRaphson procedure. The Fisher information matrix is given by $\mathbf{I}(\boldsymbol{\Delta})=\left[\mathbf{I}_{\theta_{i}, \theta_{j}}\right]_{3 \times 3}=E\left(-\frac{\partial^{2} \ell}{\partial \theta_{i} \partial \theta_{j}}\right), i, j=1,2,3$, can be numerically obtained by MATLAB or NLMIXED in SAS or R software. The total Fisher information matrix $n \mathbf{I}(\Delta)$ can be approximated by

$$
\mathbf{J}_{n}(\hat{\mathbf{\Delta}}) \approx\left[-\left.\frac{\partial^{2} \ell}{\partial \theta_{i} \partial \theta_{j}}\right|_{\Delta=\hat{\mathbf{\Delta}}}\right]_{3 X 3}, \quad i, j=1,2,3 .
$$

For a given set of observations, the matrix given in equation (26) is obtained after the convergence of the Newton-Raphson procedure via NLMIXED in SAS or R software.

\section{Monte Carlo Simulations}

In this section, the performance of the maximum likelihood estimates is examined by conducting simulation studies for different sample sizes. We conducted various simulations for different sizes sample $(n=25,50,100,200,400,800)$ via the R package. We simulate $N=1000$ samples for the true parameters values given in the Table 3 and Table 4 . The Average 
Bias and Root Mean Square Error (RMSE) were computed. The average bias and RMSE for the estimated parameter $\hat{\theta}$, say, are given by:

$$
\operatorname{ABias}(\hat{\theta})=\frac{\sum_{i=1}^{N} \hat{\theta}_{i}}{N}-\theta, \quad \text { and } \quad \operatorname{RMSE}(\hat{\theta})=\sqrt{\frac{\sum_{i=1}^{N}\left(\hat{\theta}_{i}-\theta\right)^{2}}{N}},
$$

respectively. The tables list the mean MLEs of the parameters along with the respective root mean squared errors (RMSEs) and Average Bias. We note that as the sample size $n$ increase the mean estimates of the parameters tend to be closer to the true parameter value and the RMSEs and Average Bias decay towards zero.

Table 3. Monte Carlo Simulation Results: Mean, Average Bias and RMSE

\begin{tabular}{|c|c|c|c|c|c|c|c|}
\hline \multirow[b]{2}{*}{ Parameter } & \multirow[b]{2}{*}{$n$} & \multicolumn{3}{|c|}{$\mathrm{I}(0.4,1.0,2.0)$} & \multicolumn{3}{|c|}{$\mathrm{II}(0.7,1.0,2.0)$} \\
\hline & & Mean & RMSE & Average Bias & Mean & RMSE & Average Bias \\
\hline \multirow[t]{6}{*}{$\lambda$} & 25 & 0.8527 & 0.8414 & 0.4527 & 1.1077 & 0.9361 & 0.4077 \\
\hline & 50 & 0.7165 & 0.6452 & 0.3165 & 1.0017 & 0.7799 & 0.3017 \\
\hline & 100 & 0.5987 & 0.4564 & 0.1987 & 0.8866 & 0.6092 & 0.1866 \\
\hline & 200 & 0.5292 & 0.3269 & 0.1292 & 0.8137 & 0.4638 & 0.1137 \\
\hline & 400 & 0.4845 & 0.2004 & 0.0845 & 0.7517 & 0.3008 & 0.0517 \\
\hline & 800 & 0.4727 & 0.1479 & 0.0727 & 0.7393 & 0.2120 & 0.0393 \\
\hline \multirow[t]{6}{*}{ c } & 25 & 1.3605 & 7.7538 & 0.3604 & 1.3025 & 3.0454 & 0.3025 \\
\hline & 50 & 1.2548 & 5.0278 & 0.2548 & 1.0854 & 2.4853 & 0.0854 \\
\hline & 100 & 0.9743 & 0.1172 & -0.0257 & 0.9695 & 0.1460 & -0.0304 \\
\hline & 200 & 0.9861 & 0.0789 & -0.0139 & 0.9846 & 0.0997 & -0.0153 \\
\hline & 400 & 0.9894 & 0.0551 & -0.0106 & 0.9916 & 0.0656 & -0.0083 \\
\hline & 800 & 0.9931 & 0.0374 & -0.0069 & 0.9945 & 0.0442 & -0.0054 \\
\hline \multirow[t]{6}{*}{$k$} & 25 & 1.5643 & 0.9454 & -0.4357 & 1.6391 & 1.1509 & -0.3608 \\
\hline & 50 & 1.6554 & 0.7641 & -0.3446 & 1.6532 & 0.9343 & -0.3467 \\
\hline & 100 & 1.7864 & 0.5426 & -0.2136 & 1.7736 & 0.7349 & -0.2263 \\
\hline & 200 & 1.8623 & 0.3875 & -0.1376 & 1.8607 & 0.5682 & -0.1392 \\
\hline & 400 & 1.9086 & 0.2499 & -0.0914 & 1.9267 & 0.3765 & -0.0732 \\
\hline & 800 & 1.9302 & 0.1787 & -0.0698 & 1.9515 & 0.2660 & -0.0484 \\
\hline
\end{tabular}

\section{Applications}

In this section, we present examples to illustrate the flexibility and usefulness of the LBXII distribution for data modeling. We compared the LBXII distribution with the power generalized Weibull (PGW) distribution (Bagdonavicious and Nikulin, 2002), modified weibull (MW) distribution (Lai et al., 2003), the Lindley-Weibull (LW) distribution (Asgharzadeh, Nadarajah and Sharafi, 2016), and the exponentiated power Lindley (EPL) distribution (Ghitany et al., 2013). The new model was also compared to the Weibull Exponential (WE) distribution by (Oguntunde et al., 2015) and the Marshall-Olkin Log-logistic (MOLLD) distribution (Wenhao, 2013).

The pdf of the power generalized Weibull (PGW) distribution (Bagdonavicious \& Nikulin, 2002) is given by

$$
g_{\text {PGW }}(x ; \lambda, \alpha, \beta)=\lambda \alpha \beta x^{\alpha-1}\left(1+\lambda x^{\alpha}\right)^{\beta-1} \exp \left(1-\left[1+\lambda x^{\alpha}\right]^{\beta}\right),
$$

for $\lambda, \alpha, \beta>0$. The pdf of the MW distribution is given by

$$
g_{M W}(x ; \theta, \beta \alpha)=\theta(\beta+\alpha x) x^{\beta-1} e^{\alpha x} \exp \left\{-\theta x^{\beta} e^{\alpha x}\right\}
$$

for $x>0, \lambda>0, \alpha>0$. The LW distribution (see Asgharzadeh et al., 2016), has the pdf given by

$$
g_{L W}(x ; \lambda, \alpha, \beta)=\frac{e^{-\lambda x-\alpha x^{\beta}}}{1+\lambda}\left[\lambda^{2}(1+x)+(1+\lambda+\lambda x) \alpha \beta x^{\beta-1}\right],
$$


Table 4. Monte Carlo Simulation Results: Mean, Average Bias and RMSE

\begin{tabular}{|c|c|c|c|c|c|c|c|}
\hline \multirow[b]{2}{*}{ Parameter } & \multirow[b]{2}{*}{$n$} & \multicolumn{3}{|c|}{ III $(1.5,0.7,0.3)$} & \multicolumn{3}{|c|}{ IV $(1.5,0.5,1.3)$} \\
\hline & & Mean & RMSE & Average Bias & Mean & RMSE & Average Bias \\
\hline \multirow[t]{6}{*}{$\lambda$} & 25 & 1.2528 & 0.5830 & -0.2472 & 1.2576 & 0.9319 & -0.2424 \\
\hline & 50 & 1.3205 & 0.4658 & -0.1795 & 1.3234 & 0.8359 & -0.1765 \\
\hline & 100 & 1.3837 & 0.3420 & -0.1163 & 1.3553 & 0.7269 & -0.1447 \\
\hline & 200 & 1.4238 & 0.2415 & -0.0762 & 1.3928 & 0.6168 & -0.1072 \\
\hline & 400 & 1.4540 & 0.1664 & -0.0459 & 1.4595 & 0.4449 & -0.0405 \\
\hline & 800 & 1.4754 & 0.1148 & -0.0246 & 1.4849 & 0.3179 & -0.0151 \\
\hline \multirow[t]{6}{*}{$c$} & 25 & 1.3224 & 3.4527 & 0.6224 & 0.6279 & 0.6398 & 0.1279 \\
\hline & 50 & 1.0004 & 1.4109 & 0.3004 & 0.5459 & 0.1577 & 0.0459 \\
\hline & 100 & 1.0021 & 2.8167 & 0.3021 & 0.5434 & 0.1015 & 0.0434 \\
\hline & 200 & 0.8422 & 1.2885 & 0.1422 & 0.5435 & 0.0796 & 0.0435 \\
\hline & 400 & 0.7846 & 1.3368 & 0.0846 & 0.5404 & 0.0612 & 0.0404 \\
\hline & 800 & 0.7394 & 0.4856 & 0.0394 & 0.5362 & 0.0460 & 0.0362 \\
\hline \multirow[t]{6}{*}{$k$} & 25 & 0.7159 & 0.7882 & 0.4159 & 1.1118 & 0.6807 & -0.1882 \\
\hline & 50 & 0.5865 & 0.6222 & 0.2865 & 1.1066 & 0.5895 & -0.1934 \\
\hline & 100 & 0.4888 & 0.4591 & 0.1888 & 1.1747 & 0.4652 & -0.1253 \\
\hline & 200 & 0.4253 & 0.3317 & 0.1253 & 1.2121 & 0.3596 & -0.0879 \\
\hline & 400 & 0.3679 & 0.2267 & 0.0679 & 1.2350 & 0.2417 & -0.0649 \\
\hline & 800 & 0.3399 & 0.1524 & 0.0398 & 1.2564 & 0.1710 & -0.0436 \\
\hline
\end{tabular}

for $\lambda, \alpha, \beta>0$. The EPL pdf is given by

$$
g_{E P L}(x ; \alpha, \beta, \delta)=\alpha \beta \delta x^{\alpha-1}\left(1+x^{\alpha}\right) e^{-\beta x^{\alpha}}\left[1-\left(1+\frac{\beta x^{\alpha}}{1+\beta}\right) e^{-\beta x^{\alpha}}\right]^{\delta-1},
$$

for $\alpha, \beta, \delta>0$, and $x>0$. The pdf of the WE is given by

$$
g_{W E}(x ; \alpha, \beta, \lambda)=\alpha \beta\left(\lambda e^{-\lambda x}\right)\left[\frac{\left(1-e^{-\lambda x}\right)^{\beta-1}}{\left(e^{-\lambda x}\right)^{\beta+1}}\right] e^{-\alpha\left[\frac{\left(1-e^{-\lambda x}\right)}{e^{-\lambda x}}\right]^{\beta}},
$$

for $\alpha, \beta, \lambda>0$, and the pdf of the MOLLD distribution is given by

$$
g_{\text {MOLLD }}(x ; \alpha, \beta, \lambda)=\frac{\alpha^{\beta} \beta \lambda x^{\beta-1}}{\left(x^{\beta}+\alpha^{\beta} \lambda\right)^{2}},
$$

for $\alpha, \beta, \lambda>0$ and $x>0$.

The maximum likelihood estimates (MLEs) of the LBXII parameters and its sub-models are computed by maximizing the objective function via the subroutine NLMIXED in SAS as well as the function nlm in R. The estimated values of the parameters (standard errors in parenthesis), -2log-likelihood statistic $(-2 \ln (L))$, Akaike Information Criterion $(A I C=$ $2 p-2 \ln (L))$ Bayesian Information Criterion $(B I C=p \ln (n)-2 \ln (L))$, and Consistent Akaike Information Criterion $\left(A I C C=A I C+2 \frac{p(p+1)}{n-p-1}\right)$, where $L=L(\hat{\Delta})$ is the value of the likelihood function evaluated at the parameter estimates, $n$ is the number of observations, and $p$ is the number of estimated parameters. Tables 6,8 and 10 shows results for the data sets for LBXII distribution and several non-nested models.

Plots of the fitted densities, the histogram of the data and probability plots (Chambers, Cleveland, Kleiner and Turkey, 1983) are given in Figure 6, Figure 7, and Figure 8, 9, 10 and 11, respectively. For the probability plot, we plotted $G_{L B X I I}\left(x_{(j)} ; \hat{\lambda}, \hat{c}, \hat{k}\right)$ against $\frac{j-0.375}{n+0.25}, j=1,2, \cdots, n$, where $x_{(j)}$ are the ordered values of the observed data. The measures 
of closeness are given by the sum of squares

$$
S S=\sum_{j=1}^{n}\left[G_{L B X I I}\left(x_{(j)}\right)-\left(\frac{j-0.375}{n+0.25}\right)\right]^{2} .
$$

The goodness-of-fit statistics $W^{*}$ and $A^{*}$, described by (Chen and Balakrishnan, 1995) are presented in the tables. The Kolmogorov-Smirnov (K-S) statistic and its p-value are also presented. These statistics can be used to verify which distribution fits better to the data. In general, the smaller the values of $W^{*}, A^{*}$ and K-S, the better the fit.

\subsection{Failure Times of 50 Components Data}

The first data set consists of failure times of 50 components (per 1000h) taken from (Murthy, Xie and Jiang 2004).

Table 5. Failure times of 50 components data

\begin{tabular}{ccccccccccccc}
\hline & & & & & & & & & & & & \\
0.036 & 0.148 & 0.590 & 3.076 & 6.816 & 0.058 & 0.183 & 0.618 & 3.147 & 7.896 & 0.061 & 0.192 & 0.645 \\
3.625 & 7.904 & 0.074 & 0.254 & 0.961 & 3.704 & 8.022 & 0.078 & 0.262 & 1.228 & 3.931 & 9.337 & 0.086 \\
0.379 & 1.600 & 4.073 & 10.940 & 0.102 & 0.381 & 2.006 & 4.393 & 11.020 & 0.103 & 0.538 & 2.054 & 4.534 \\
13.880 & 0.114 & 0.570 & 2.804 & 4.893 & 14.73 & 0.116 & 0.574 & 3.058 & 6.274 & 15.08 & & \\
\hline
\end{tabular}

The estimated asymptotic variance-covariance matrix of the maximum likelihood estimates for LBXII distribution for the failure times of 50 components data are as follows:

$$
\left[\begin{array}{ccc}
0.0035 & -2.6786 \times 10^{-3} & -4.5626 \times 10^{-3} \\
-0.0027 & 1.6200 \times 10^{-2} & -3.6119 \times 10^{-6} \\
-0.0046 & -3.6119 \times 10^{-6} & 2.7161 \times 10^{-2}
\end{array}\right]
$$

The approximate $95 \%$ confidence intervals for the parameters, $\lambda, c$ and $k$ are $(0.2094 \pm 1.96 \times \sqrt{0.0035})$, $\left(0.7389 \pm 1.96 \times \sqrt{1.6200 \times 10^{-2}}\right)$ and $\left(0.7305 \pm 1.96 \times \sqrt{2.7161 \times 10^{-2}}\right)$, respectively.

For the failure times of 50 components data, the values of the statistic AIC, AICC and BIC are the smallest for the LBXII distribution. Also the goodness of fit statistics $W^{*}, A^{*}$ and $K-S$, are the lowest. The P-value and the $S S$ values from the probability plots indicate that indeed our model performs better than the non-nested models that were considered. 
Fitted PDF

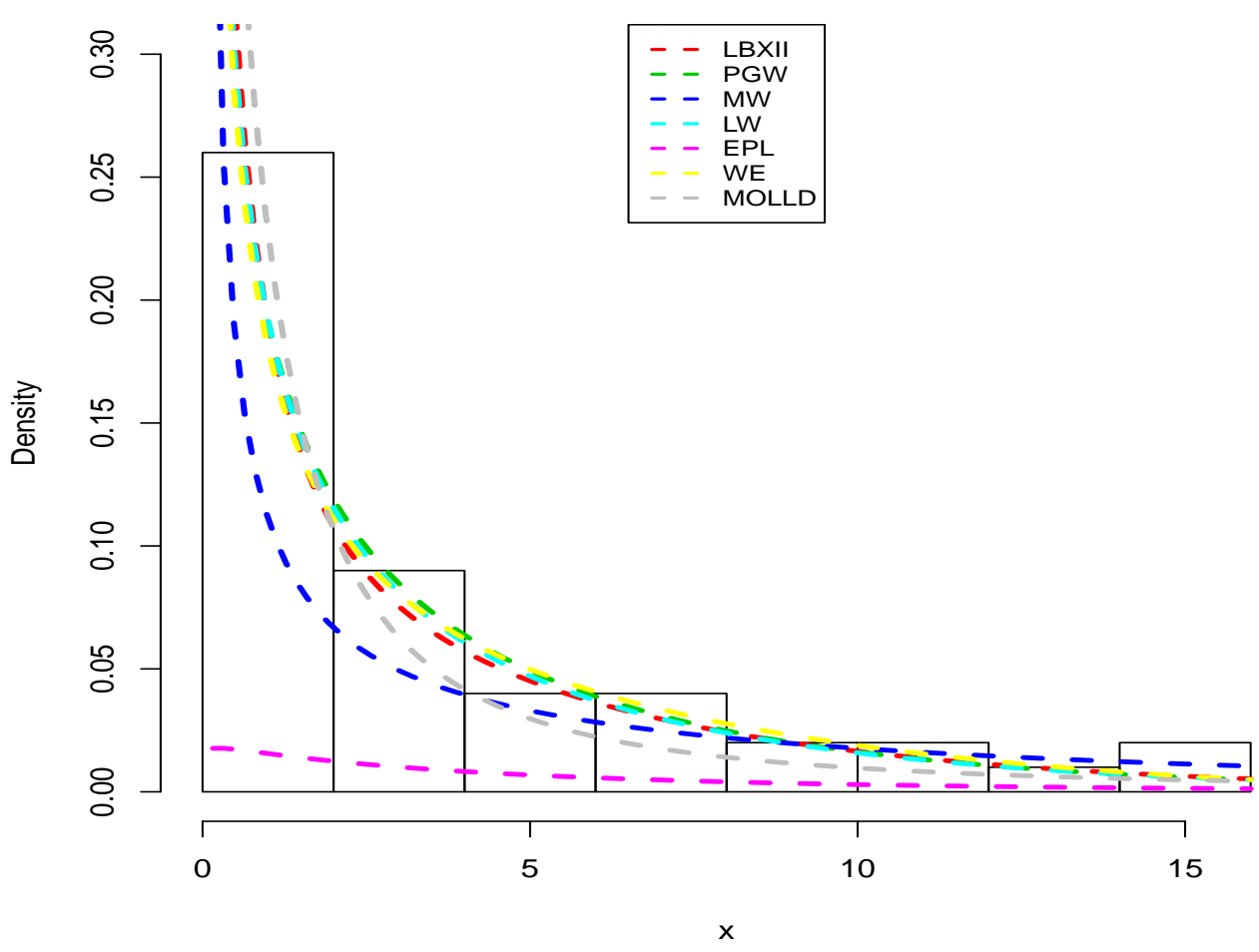

Figure 6. Fitted pdf's for failure times data

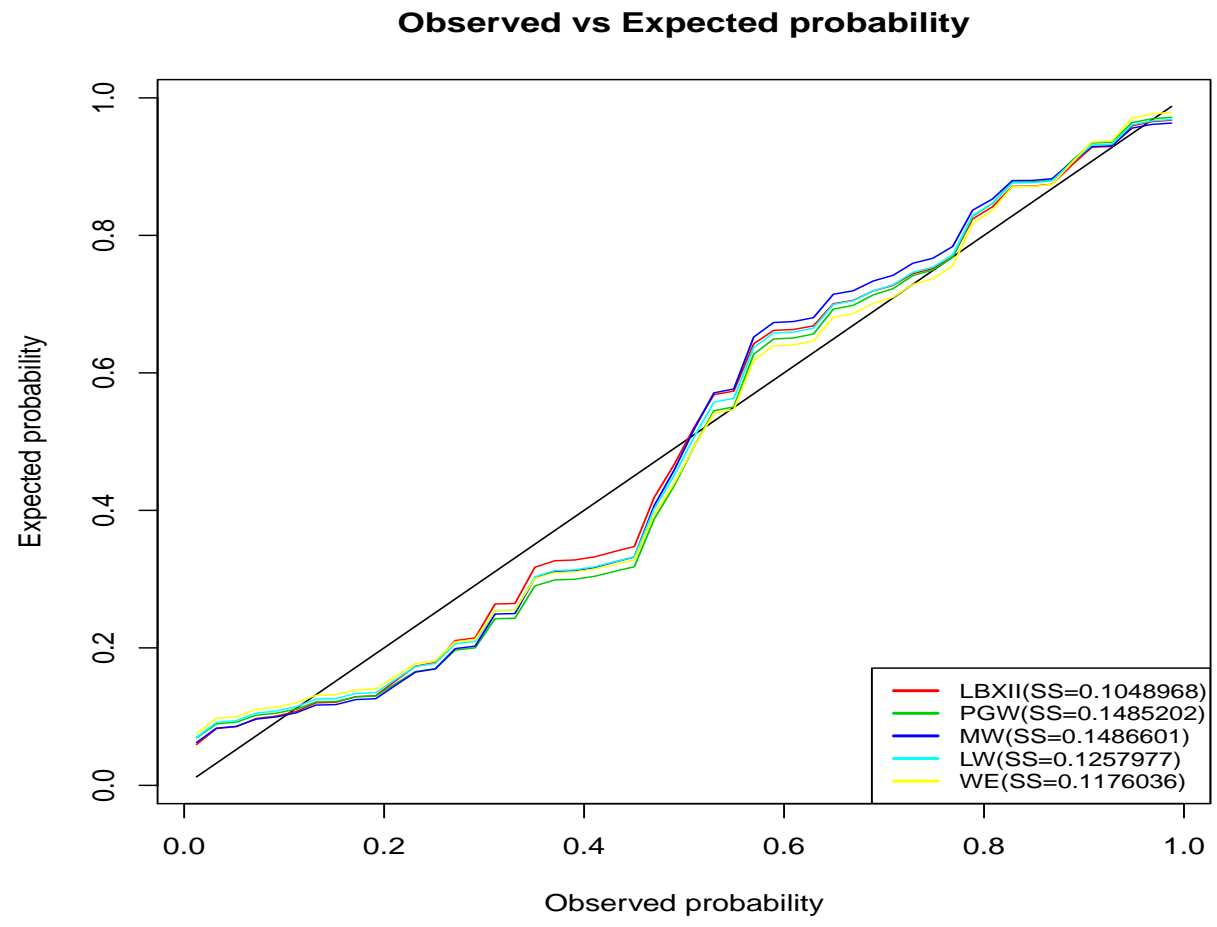

Figure 7. Probability plots for failure times data 
Table 6. Failure times of 50 components data

\begin{tabular}{|c|c|c|c|c|c|c|c|c|c|c|c|c|}
\hline & \multicolumn{4}{|c|}{ Estimates } & \multicolumn{4}{|c|}{ Statistics } & \multirow[b]{2}{*}{$A^{*}$} & \multirow[b]{2}{*}{$K-S$} & \multirow[b]{2}{*}{$P$-value } & \multirow[b]{2}{*}{$S S$} \\
\hline Model & $\lambda$ & $c$ & $k$ & $-2 \log L$ & $A I C$ & AICC & $B I C$ & $W^{*}$ & & & & \\
\hline LBXII & $\begin{array}{c}0.2094 \\
(0.0595) \\
\lambda\end{array}$ & $\begin{array}{c}0.7388 \\
(0.1272) \\
\alpha\end{array}$ & $\begin{array}{c}0.7305 \\
(0.1648) \\
\beta\end{array}$ & 200.7 & 206.7 & 207.2 & 212.4 & 0.1153 & 0.7431 & 0.1126 & 0.5141 & 0.1049 \\
\hline PGW & $\begin{array}{c}0.1261 \\
(0.5100) \\
\theta\end{array}$ & $\begin{array}{c}0.5477 \\
(0.1841) \\
\beta\end{array}$ & $\begin{array}{c}3.4268 \\
(12.7544) \\
\alpha\end{array}$ & 204.4 & 210.9 & 210.9 & 216.2 & 0.1446 & 0.9276 & 0.1419 & 0.2418 & 0.1485 \\
\hline MW & $\begin{array}{c}0.0439 \\
(0.1315) \\
\lambda \\
\end{array}$ & $\begin{array}{c}0.6195 \\
(0.1545) \\
\alpha \\
\end{array}$ & $\begin{array}{c}0.4925 \\
(0.1808) \\
\beta \\
\end{array}$ & 204.6 & 210.6 & 211.2 & 216.4 & 0.1502 & 0.9452 & 0.1279 & 0.3564 & 0.1487 \\
\hline LW & $\begin{array}{c}0.1325 \\
(0.0950) \\
\alpha\end{array}$ & $\begin{array}{c}0.5055 \\
(0.1089) \\
\beta\end{array}$ & $\begin{array}{c}0.5863 \\
(0.1068) \\
\delta\end{array}$ & 203.6 & 209.6 & 210.2 & 215.4 & 0.1385 & 0.8862 & 0.1278 & 0.3563 & 0.1258 \\
\hline EPL & $\begin{array}{c}0.5632 \\
(0.0598) \\
\alpha\end{array}$ & $\begin{array}{c}0.5290 \\
(0.0682) \\
\beta\end{array}$ & $\begin{array}{c}0.5094 \\
(0.0499) \\
\delta\end{array}$ & 462.5 & 468.5 & 469.0 & 474.2 & 0.4229 & 2.6352 & 0.58409 & $8.8 \times 10^{-16}$ & 3.6252 \\
\hline WE & $\begin{array}{c}1.6476 \\
(1.0097) \\
\alpha\end{array}$ & $\begin{array}{c}0.5544 \\
(0.0942) \\
\beta\end{array}$ & $\begin{array}{c}0.1149 \\
(0.0807) \\
\lambda\end{array}$ & 202.4 & 308.8 & 309.3 & 314.7 & 0.16408 & 1.0574 & 0.3672 & $1.49 \times 10^{-06}$ & 0.1176 \\
\hline MOLLD & $\begin{array}{c}1.0619 \\
(131.8401)\end{array}$ & $\begin{array}{c}0.9108 \\
(0.1033)\end{array}$ & $\begin{array}{c}1.0603 \\
(119.8942)\end{array}$ & 210.9 & 234.3 & 234.8 & 240.1 & 0.2480 & 1.5451 & 0.3109 & $8.607 \times 10^{-05}$ & 6.9697 \\
\hline
\end{tabular}

\subsection{Epoxy Strands Failure at 90\% Stress Level Data}

The second data set represent the stress-rupture life of kevlar 49/epoxy strands which were subjected to constant sustained pressure at the $90 \%$ stress level until all had failed, so that we have complete data with exact times of failure, given by (Barlow, Toland and Freeman, 1984) and (Andrews and Herzberg, 1985).

Table 7. Epoxy strands failure at $90 \%$ stress level data

\begin{tabular}{llllllllllllllll}
\hline 0.01 & 0.01 & 0.02 & 0.02 & 0.02 & 0.03 & 0.03 & 0.04 & 0.05 & 0.06 & 0.07 & 0.07 & 0.08 & 0.09 & 0.09 \\
0.10 & 0.10 & 0.11 & 0.11 & 0.12 & 0.13 & 0.18 & 0.19 & 0.20 & 0.23 & 0.24 & 0.24 & 0.29 & 0.34 & 0.35 \\
0.36 & 0.38 & 0.40 & 0.42 & 0.43 & 0.52 & 0.54 & 0.56 & 0.60 & 0.60 & 0.63 & 0.65 & 0.67 & 0.68 & 0.72 \\
0.72 & 0.72 & 0.73 & 0.79 & 0.79 & 0.80 & 0.80 & 0.83 & 0.85 & 0.90 & 0.92 & 0.95 & 0.99 & 1.00 & 1.01 \\
1.02 & 1.03 & 1.05 & 1.10 & 1.10 & 1.11 & 1.15 & 1.18 & 1.20 & 1.29 & 1.31 & 1.33 & 1.34 & 1.40 & 1.43 \\
1.45 & 1.50 & 1.51 & 1.52 & 1.53 & 1.54 & 1.54 & 1.55 & 1.58 & 1.60 & 1.63 & 1.64 & 1.80 & 1.80 & 1.81 \\
2.02 & 2.05 & 2.14 & 2.17 & 2.33 & 3.03 & 3.03 & 3.34 & 4.20 & 4.69 & 7.89 & & & & \\
\hline
\end{tabular}

The estimated variance-covariance matrix for the LBXII of the Epoxy strands failure at 90\% stress level data is given by

$$
\left[\begin{array}{ccc}
0.0401 & -0.0236 & -0.0459 \\
-0.0236 & 0.0446 & 0.0357 \\
-0.0459 & 0.0357 & 0.0749
\end{array}\right]
$$

The $95 \%$ confidence intervals for the parameters $\lambda, c$ and $k$ of LBXII (Epoxy strands failure at 90\% stress level data) are: $(1.1187 \pm 1.96 \times \sqrt{0.0401}),(0.7219 \pm 1.96 \times \sqrt{0.0446})$ and $(0.3952 \pm 1.96 \times \sqrt{0.0749})$, respectively.

For epoxy strands failure at $90 \%$ stress level data, the values of the AIC, AICC and BIC are smaller for the LBXII distribution also the values of the statistics $W^{*}, A^{*}, K-S$ and its P-value, and the $S S$ value indicate that the LBXII distribution performs better than all the models as indicated in Table 8 . 
Table 8. Epoxy strands failure at $90 \%$ stress level data

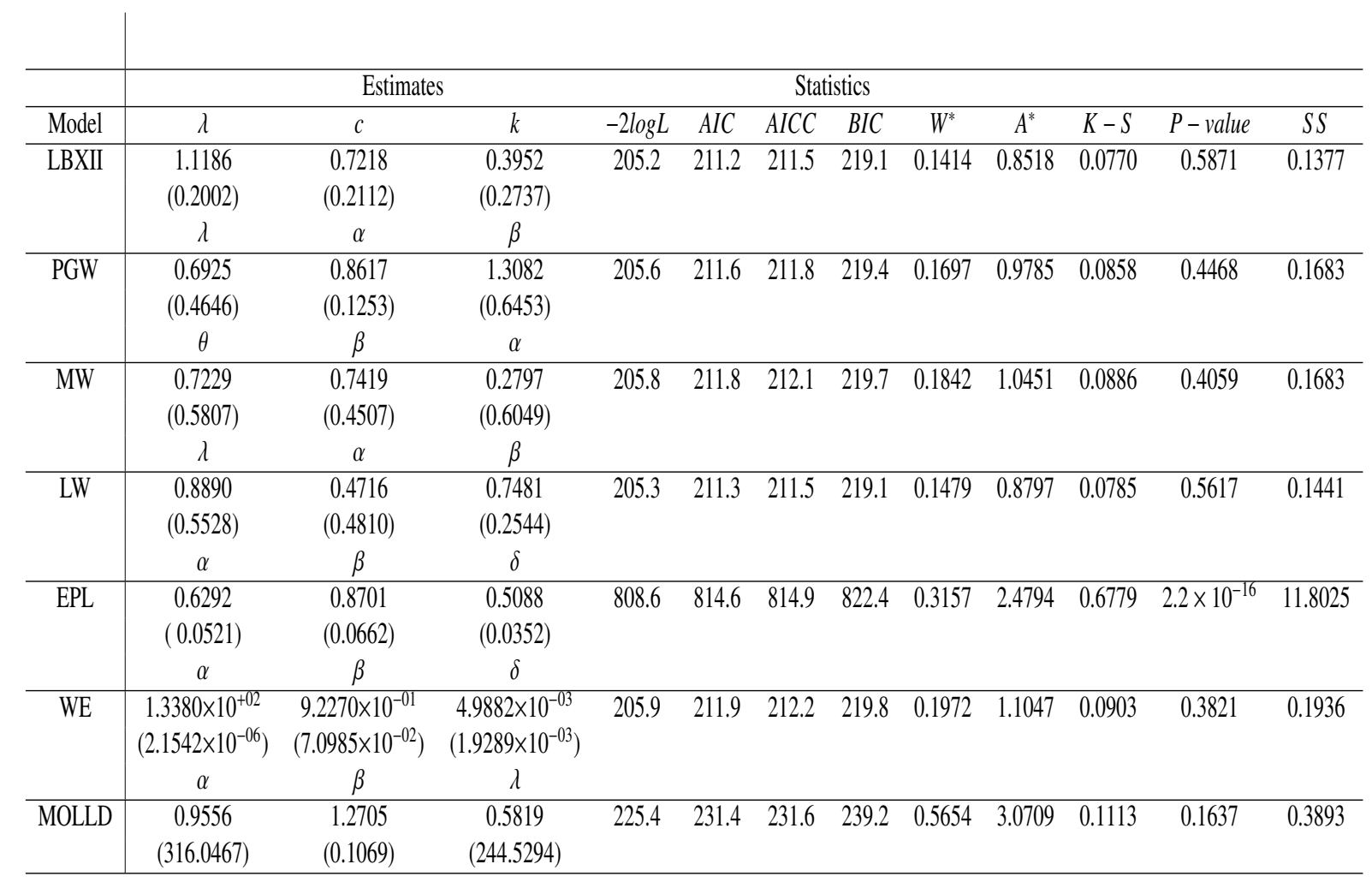

\section{Fitted PDF}

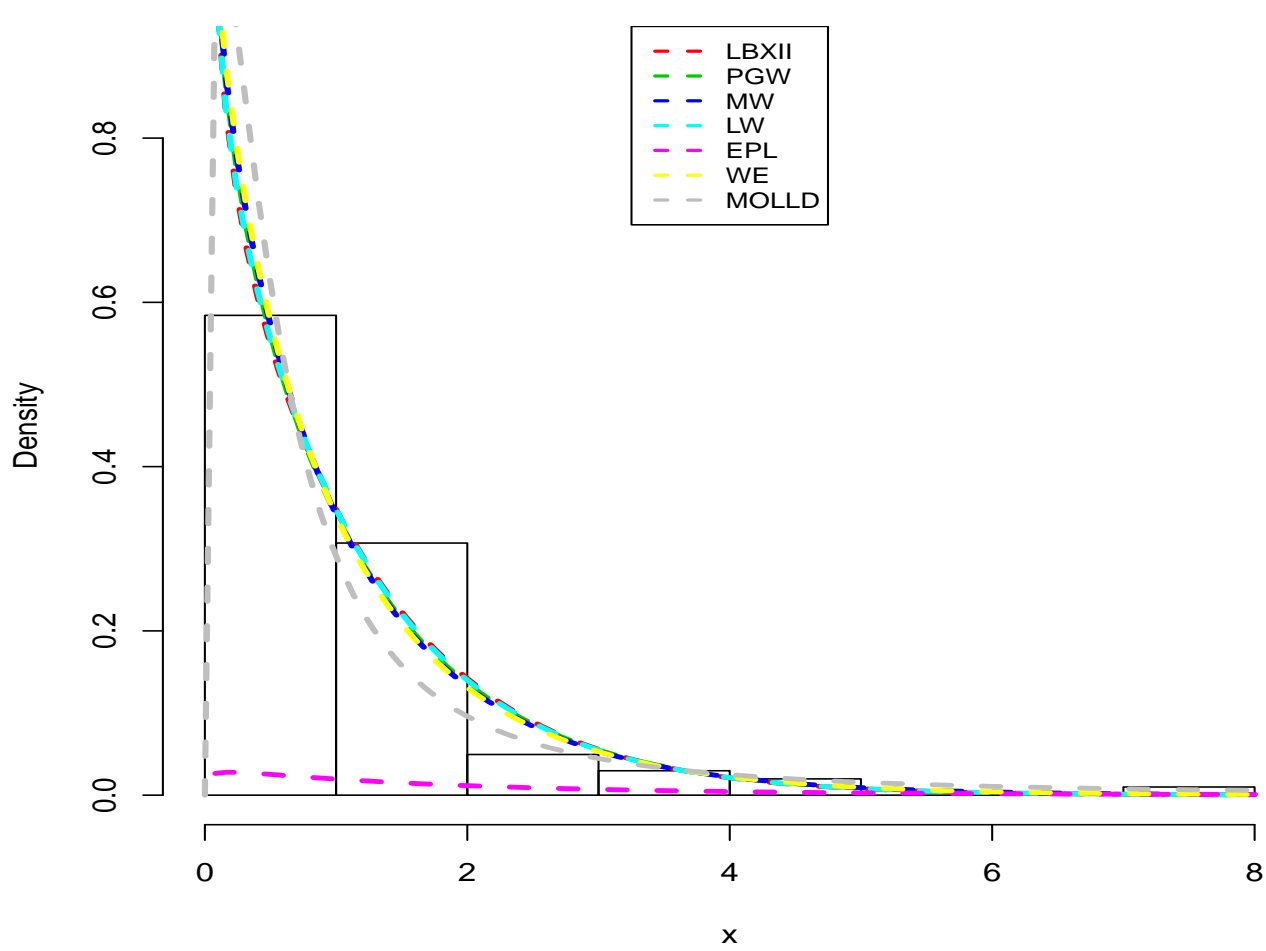

Figure 8. Fitted pdf's for epoxy strands data 


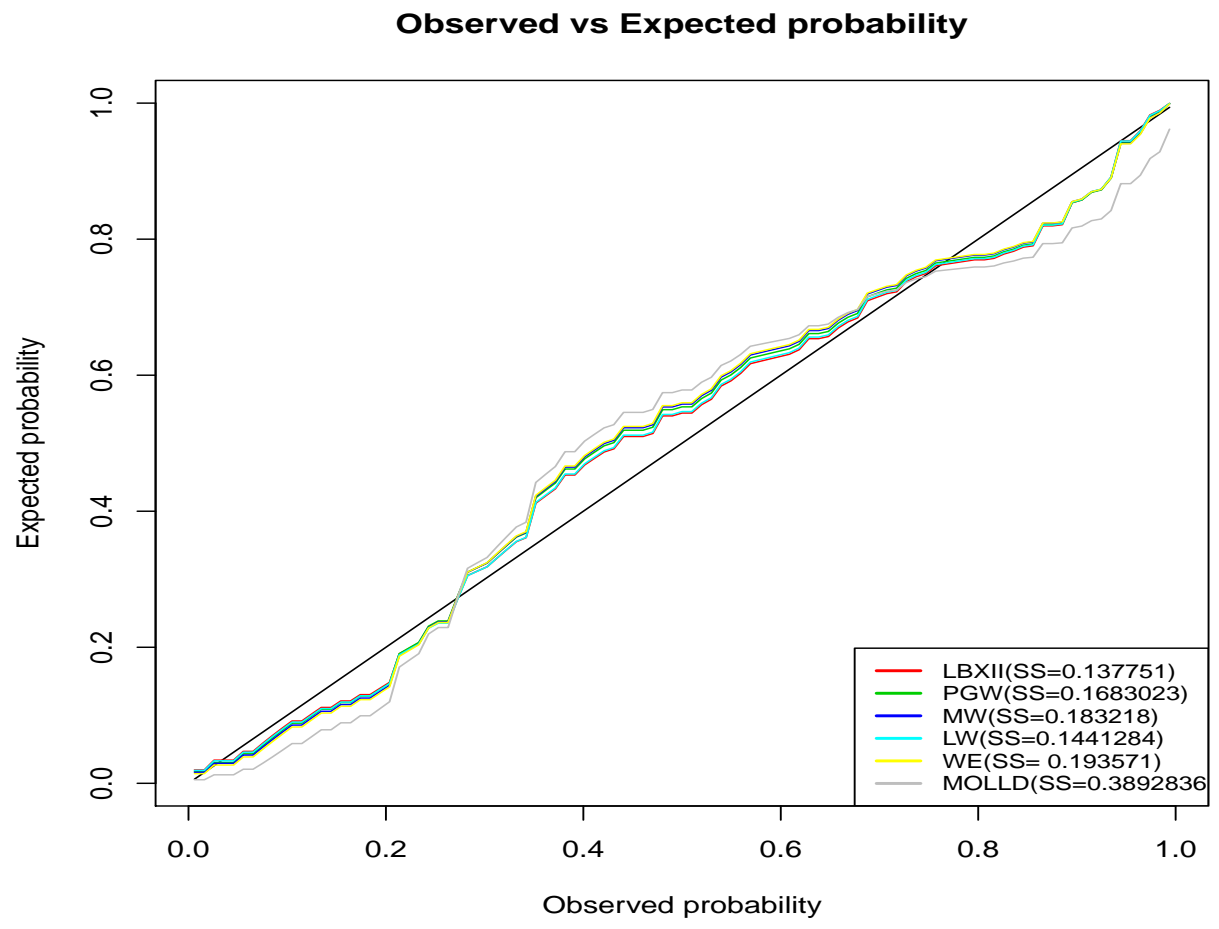

Figure 9. Probability plots for epoxy strands data

\subsection{Wheaton River Data}

The third data set are the exceedances of flood peaks (in m3/s) of the Wheaton River near Carcross in Yukon Territory, Canada. The data consist of 72 exceedances for the years 1958-1984. These data were also analyzed by (Akinsete, Famoye and Lee, 2008).

Table 9. Wheaton River data set

\begin{tabular}{ccccccccccccccccc}
\hline & & & & & & & & & & & & & & & & \\
1.7 & 2.2 & 14.4 & 1.1 & 0.4 & 20.6 & 5.3 & 0.7 & 1.9 & 13.0 & 12.0 & 9.3 & 1.4 & 18.7 & 8.5 & 25.5 \\
11.6 & 14.1 & 22.1 & 1.1 & 2.5 & 14.4 & 1.7 & 37.6 & 0.6 & 2.2 & 39.0 & 0.3 & 15.0 & 11.0 & 7.3 & 22.9 \\
1.7 & 0.1 & 1.1 & 0.6 & 9.0 & 1.7 & 7.0 & 20.1 & 0.4 & 2.8 & 14.1 & 9.9 & 10.4 & 10.7 & 30.0 & 3.6 \\
5.6 & 30.8 & 13.3 & 4.2 & 25.5 & 3.4 & 11.9 & 21.5 & 27.6 & 36.4 & 2.7 & 64.0 & 1.5 & 2.5 & 27.4 & \\
1.0 & 27.1 & 20.2 & 16.8 & 5.3 & 9.7 & 27.5 & 2.5 & 27.0 & & & & & & & \\
\hline
\end{tabular}

The estimated asymptotic variance-covariance matrix of the maximum likelihood estimates for LBXII distribution for Wheaton River data set are as follows:

$$
\left[\begin{array}{ccc}
0.0002 & -0.0009 & -0.0002 \\
-0.0009 & 0.1567 & -0.0125 \\
-0.0002 & -0.0125 & 0.0024
\end{array}\right]
$$

The approximate $95 \%$ confidence intervals for the parameters, $\lambda, c$ and $k$ are $(0.0923 \pm 1.96 \times \sqrt{0.0002}),(1.4905 \pm 1.96 \times \sqrt{0.1567})$ and $(0.1565 \pm 1.96 \times \sqrt{0.0024})$, respectively. 
Table 10. Wheaton River data set

\begin{tabular}{|c|c|c|c|c|c|c|c|c|c|c|c|c|}
\hline \multirow[b]{2}{*}{ Model } & \multicolumn{4}{|c|}{ Estimates } & \multicolumn{4}{|c|}{ Statistics } & \multirow[b]{2}{*}{$A^{*}$} & \multirow[b]{2}{*}{$K-S$} & \multirow[b]{2}{*}{$P$-value } & \multirow[b]{2}{*}{$S S$} \\
\hline & $\lambda$ & $c$ & $k$ & $-2 \log L$ & $A I C$ & $A I C C$ & $B I C$ & $W^{*}$ & & & & \\
\hline LBXII & $\begin{array}{c}0.0923 \\
(0.0146) \\
\lambda\end{array}$ & $\begin{array}{c}1.4905 \\
(0.3959) \\
\alpha\end{array}$ & $\begin{array}{c}0.1565 \\
(0.0494) \\
\beta\end{array}$ & 497.5 & 503.5 & 503.8 & 510.3 & 0.0771 & 0.4348 & 0.0757 & 0.8039 & 0.0779 \\
\hline PGW & $\begin{array}{c}0.0365 \\
(0.0607) \\
\theta\end{array}$ & $\begin{array}{c}0.7441 \\
(0.1472) \\
\beta\end{array}$ & $\begin{array}{c}3.2248 \\
(5.5776) \\
\alpha\end{array}$ & 501.8 & 507.8 & 508.2 & 514.7 & 0.1041 & 0.6326 & 0.1063 & 0.3903 & 0.1059 \\
\hline MW & $\begin{array}{c}0.0412 \\
(0.1016) \\
\alpha\end{array}$ & $\begin{array}{c}0.8120 \\
(0.4037) \\
\beta\end{array}$ & $\begin{array}{c}0.0704 \\
(0.1046) \\
\delta\end{array}$ & 502.9 & 507.3 & 507.74 & 514.2 & 0.0969 & 0.5889 & 0.0994 & 0.4752 & 0.14500 \\
\hline LW & $\begin{array}{c}0.0468 \\
(0.0398) \\
\alpha\end{array}$ & $\begin{array}{c}0.1176 \\
(0.0342) \\
\beta\end{array}$ & $\begin{array}{c}0.8027 \\
(0.1557) \\
\delta\end{array}$ & 502.2 & 508.2 & 508.6 & 515.1 & 0.1203 & 0.7038 & 0.1017 & 0.4458 & 0.1177 \\
\hline EPL & $\begin{array}{c}1.3251 \\
(0.0938) \\
\alpha\end{array}$ & $\begin{array}{c}0.0301 \\
(0.0091) \\
\beta\end{array}$ & $\begin{array}{c}0.5102 \\
(0.0417) \\
\delta\end{array}$ & 665.3 & 671.3 & 671.6 & 678.1 & 0.4971 & 3.1751 & 0.1843 & 0.0150 & 0.8055 \\
\hline WE & $\begin{array}{c}2.3899 \\
(2.1029) \\
\alpha\end{array}$ & $\begin{array}{c}0.7808 \\
(0.1181) \\
\beta\end{array}$ & $\begin{array}{c}0.0227 \\
(0.0181) \\
\lambda\end{array}$ & 501.3 & 507.3 & 507.7 & 514.2 & 0.0969 & 0.5889 & 0.0994 & 0.4752 & 0.0946 \\
\hline MOLLD & $\begin{array}{c}2.3411 \\
(0.3769)\end{array}$ & $\begin{array}{c}1.2127 \\
(0.1167)\end{array}$ & $\begin{array}{c}3.6168 \\
(0.2012)\end{array}$ & 515.7 & 521.8 & 522.0 & 528.5 & 0.2961 & 1.6484 & 0.1138 & 0.309 & 0.2328 \\
\hline
\end{tabular}

\section{Fitted PDF}

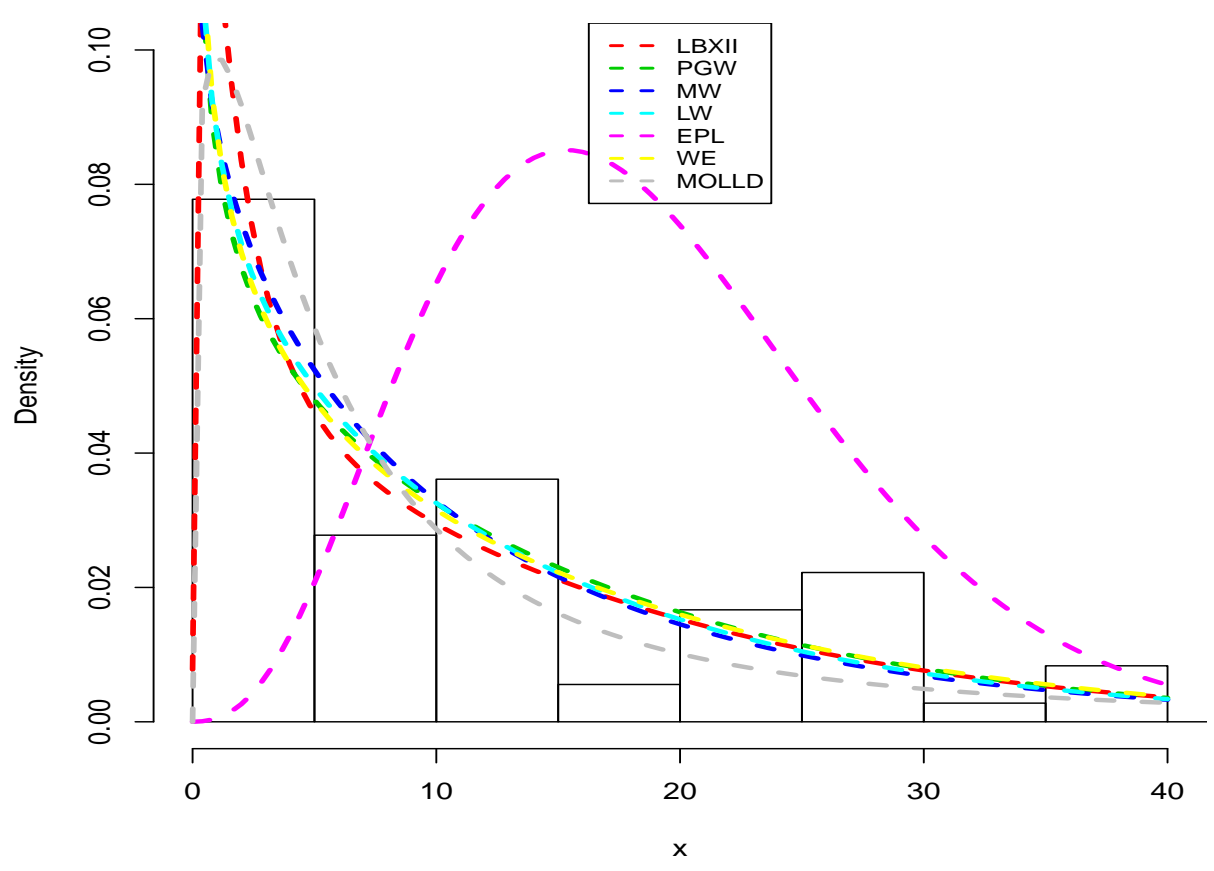

Figure 10. Fitted pdf's for Wheaton River data

Similarly for Wheaton River data, the values of the statistics $K-S$, P-value, and $S S$ from the probability plots shows that the LBXII distribution performs better for this data set. In overall the LBXII distribution performs better PGW, MW, EPL, LW, WE and the MOLLD distributions for the Wheaton River data. 


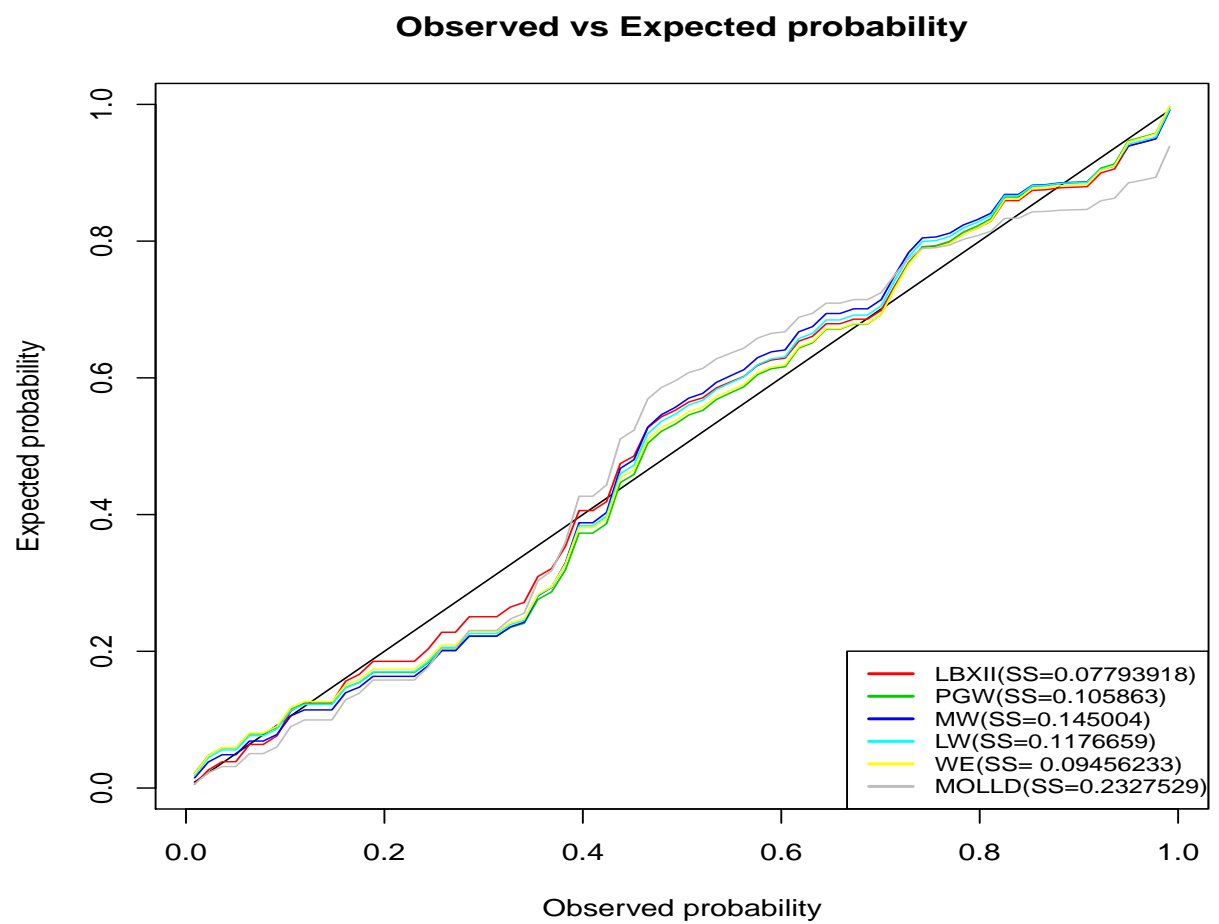

Figure 11. Probability plots for Wheaton River data

\section{Concluding Remarks}

A new three parameter distribution called the Lindley-Burr XII (LBXII) distribution is presented, which extends the Burr XII (BXII) distribution. The LBXII distribution has two components namely the Lindley and the Burr XII distributions. The LBXII distribution possesses hazard function with flexible behaviour. We derive expressions for the moments, mean and median deviations, distribution of order statistics and entropy. Maximum likelihood estimation technique is used to estimate the model parameters. The performance of the LBXII distribution was examined by conducting various simulations for different sizes. Finally, the distribution was fitted to real data sets to illustrate empirically that the LBXII provides consistently better fits to two real data sets than other six non-nested distributions with same number of parameters. The two examples proves that the LBXII is a desired alternative for modelling survival data. The LBXII include several other distributions, hence, it is our belief that the LBXII will attract wider applications in several different areas of research.

\section{Acknowledgments}

The authors are grateful to the referees and the editors for their comments which greatly improved the organisation of this paper.

\section{References}

Akinsete, A., Famoye, F., \& Lee, C. (2008). The beta-Pareto distribution. Statistics, 42(6), 547-563. https://doi.org/10.1080/02331880801983876

Andrews, D. F., \& Herzberg, A. M. (1985). Data: A Collection of Problems from Many Fields for the Student and Research Worker. Springer, New York.

Asgharzadeh, A., Nadarajah, S., \& Sharafi, F. (2016). Weibull Lindley Distribution. Revstat-Statistical Journal, 16(1), $87-113$

Bagdonavicius, V., \& Nikulin, M. (2002). Accelerated Life Model. Modeling and Statistical Analysis, Chapman and Hall/CRC. https://doi.org/10.1201/9781420035872

Barlow, R. E., Toland, R. H., \& Freeman, T. (1984). A Bayesian Analysis of Stress-Rupture Life of Kevlar 49/epoxy Spherical Pressure Vessels. In: Proceeding of Canadian Conference in Applied Statistics, Marcel Dekker, New York.

Chambers, J., Cleveland, W., Kleiner, B., \& Tukey, J. (1983). Graphical Methods for Data Analysis. Chapman and Hall, 
London.

Chen, G., \& Balakrishnan, N. (1995). A General Purpose Approximate Goodness-of-fit Test. Journal of Quality Technology, 27(2), 154-161. https://doi.org/10.1080/00224065.1995.11979578.

Ghitany, M. E., Al-Mutairi, D. K., Balakrishnan, N., \& Al-Enezi, L. J. (2013). Power Lindley Distribution and Associated Inference, Computational Statistics $\mathcal{E}$ Data Analysis, 64, 20-33. https://doi.org/10.1016/j.csda.2013.02.026.

Lai, C. D., Xie, M., \& Murthy, D. N. P. (2003). A Modified Weibull distribution. IEEE Transactions on Reliability, 52(1), 33-37. https://doi.org/10.1109/TR.2002.805788

Lindley, D. V. (1958). Fiducial Distributions and Bayes Theorem Journal of the Royal Statistical Society Series B., 20(1), 102-107. https://doi.org/10.1111/j.2517-6161.1958.tb00278.x

Murthy, D. P., Xie, M., \& Jiang, R. (2004). Weibull Models, John Wiley and Sons, 505.

Nadarajah, S., Bakouch, H. S., \& Tahmasbi, R. (2011). A Generalized Lindley Distribution, Sankhya B, 73, $331-359$. https://doi.org/10.1007/s13571-011-0025-9.

Oguntunde, P. E., Balogun, O. S., Okagbue, H. I., \& Bishop, S. A. (2015). The Weibull-Exponential Distribution: Its Properties and Applications, Journal of Applied Sciences, 15 (11), 1305-1311. DOI: 10.3923/jas.2015.1305.1311.

Oluyede, B. O., \& Yang, T. (2015). A New Class of Generalized Lindley Distributions with Applications, Journal of Statistical Computation and Simulation, 85(10), 2072-2100.

Oluyede, B. O., Yang, T., \& Omolo, B. (2015). A Generalized Class of Kumaraswamy Lindley Distribution with Applications to Lifetime Data, Journal of Computations $\mathcal{F}$ Modelling, 5(1), 27-70.

Oluyede, B. O., Foya, S., Warahena-Liyanage, G., \& Huang, S. (2016). The Log-logistic Weibull Distribution with Applications to Lifetime Data, Austrian Journal of Statistics, 45(3), 43-69. https://doi.org/10.17713/ajs.v45i3.107

Rényi, A. (1960). On Measures of Entropy and Information, Proceedings of the Fourth Berkeley Symposium on Mathematical Statistics and Probability, 1, 547-561. https://projecteuclid.org/euclid.bsmsp/1200512181.

Wenhao, G. (2013). Marshall-Olkin Extended Log-Logistic Distribution and its Application in Minication Processes, Applied Mathematical Sciences, 7(80), 3947-3961. https://doi.org/10.12988/ams.2013.35268

Zakerzadeh, H., \& Dolati, A. (2009). Generalized Lindley Distribution. Journal of Mathematical Extension, 3(2), 13-25.

\section{Copyrights}

Copyright for this article is retained by the author(s), with first publication rights granted to the journal.

This is an open-access article distributed under the terms and conditions of the Creative Commons Attribution license (http://creativecommons.org/licenses/by/4.0/). 